\title{
Balanced Realizations of Symmetric Composite Systems
}

\author{
James Lam ${ }^{1}$ \\ Department of Mechanical Engineering \\ University of Hong Kong \\ Pokfulam Road, HONG KONG
}

\author{
Guang-Hong Yang \\ School of Electrical and Electronic Engineering \\ The Nanyang Technological University \\ Nanyang Avenue, SINGAPORE 639798
}

\begin{abstract}
In this paper, we investigate the structural properties of balanced realizations, and their truncation, of a class of systems with symmetric interconnected and identical structure. It is established that the balanced realizations of such symmetric composite systems can be constructed via the balanced realizations of two auxiliary systems of comparatively low dimensions. The corresponding truncated realizations, which also have a certain symmetric structure, are explicitly constructed.
\end{abstract}

\section{Introduction}

In many naturally evolving large-scale systems such as biological systems, social systems and management systems, a frequently observed fundamental feature is that some subsystems commonly possess similar structure. This also occurs widely in engineering systems, for example in the stabilization problem for a power system in a plant with identical units [AC91], the voltage control of the feeding nodes in an electric power system consisting of connected synchronous machines [BL88], and the control design of industrial manipulators with several degrees of freedom [VS82]. More recently, the output regulation problem for this class of large-scale systems was investigated [Liu92] (see [YZ95b] for more references). From the modelling and synthesis point of view, such a large-scale system may contain weakly controllable and weakly observable modes that do not contribute much to the input-output characteristics of the system. To reduce the effort of computation as well as controller implementation, it is necessary to look for low-order representations of these systems. In particular, we would like to consider reduced-order models which preserve the symmetrically interconnected structure.

Balanced realization truncation has been an important technique for model reduction of stable systems. It was first introduced to the control engineering field by Moore [Moo81]. The underlying ideas involve Principal Component Analysis which has been widely used in statistical analysis and Kalman's controllable/observable canonical realization of a system. The method was further analyzed by Pernebo and Silverman [PS82]. Apart from the apparent successes in many practical engineering applications, the method is also theoretically appealing due to the existence of a prior error bounds calculated based

\footnotetext{
${ }^{1}$ This work has been supported by RGC Grant HKU 544/96E
}

on the Hankel singular values of the full order model [Glo84,LA92].

In this paper, we study the construction of balanced realizations of symmetric composite systems. In particular, we exploit their symmetric interconnected structure in the balancing procedure which significantly reduce the effort of computation. This paper is divided into four sections. The mathematical description of the symmetric composite systems and preliminary results are given in Section 2. In Section 3, the construction of a balanced realization of a symmetric composite system via the balancing of two modified subsystems is described. Also, the structure of its truncation is studied. Finally, conclusions are given in Section 4.

\section{Model Description and Preliminary Results}

Consider a finite-dimensional linear time-invariant interconnected system which is composed of $N(N>1)$ identical subsystems with the overall state-space description given by

$$
\begin{aligned}
\dot{x} & =\mathcal{A} x+\mathcal{B} u \\
y & =\mathcal{C} x
\end{aligned}
$$

where

$$
\begin{aligned}
x & =\left[\begin{array}{lll}
x_{1}^{T} & \cdots & x_{N}^{T}
\end{array}\right]^{T}, \quad x_{i} \in \mathbb{R}^{n} \\
u & =\left[\begin{array}{lll}
u_{1}^{T} & \cdots & u_{N}^{T}
\end{array}\right]^{T}, \quad u_{i} \in \mathbb{R}^{m} \\
y & =\left[\begin{array}{lll}
y_{1}^{T} & \cdots & y_{N}^{T}
\end{array}\right]^{T}, \quad y_{i} \in \mathbb{R}^{p}
\end{aligned}
$$

in which $u_{i}, x_{i}$, and $y_{i}$ are the input, state, and output vector of the $i$ th subsystem respectively, and the composite state matrix $\mathcal{A} \in \mathbb{R}^{N n \times N n}$, input matrix $\mathcal{B} \in \mathbb{R}^{N n \times N m}$, and output matrix $\mathcal{C} \in \mathbb{R}^{N_{p \times N n}}$ have the following structure:

$$
\begin{aligned}
\mathcal{A} & =\left[\begin{array}{cccc}
A & A_{0} & \cdots & A_{0} \\
A_{0} & A & \ddots & \vdots \\
\vdots & \ddots & \ddots & A_{0} \\
A_{0} & \cdots & A_{0} & A
\end{array}\right] \\
\mathcal{B} & =\left[\begin{array}{cccc}
B & B_{0} & \cdots & B_{0} \\
B_{0} & B & \ddots & \vdots \\
\vdots & \ddots & \ddots & B_{0} \\
B_{0} & \cdots & B_{0} & B
\end{array}\right]
\end{aligned}
$$




$$
\mathcal{C}=\left[\begin{array}{cccc}
C & C_{0} & \cdots & C_{0} \\
C_{0} & C & \ddots & \vdots \\
\vdots & \ddots & \ddots & C_{0} \\
C_{0} & \cdots & C_{0} & C
\end{array}\right]
$$

The realization $(\mathcal{A}, \mathcal{B}, \mathcal{C})$ is assumed to be minimal. This model was first proposed by Lunze in [Lun86] where he investigated a number of its qualitative properties. Some recent extensive studies on the class of systems are referred [SE91,YZ95a].

Let $v_{0}, v_{1}, \ldots, v_{N-1}$ denote the $N$ th roots of unity,

$$
v_{j}=\exp (2 \pi j \sqrt{-1} / N) \quad, \quad j=0,1, \ldots, N-1
$$

and let

$$
m_{j}=\left[\begin{array}{lllll}
1 & v_{j} & v_{j}^{2} & \cdots & v_{j}^{N-1}
\end{array}\right]^{T}, \quad j=0,1, \ldots, N-1
$$

Denote

$$
\ell= \begin{cases}\frac{N-1}{2} & \text { if } N \text { is odd } \\ \frac{N^{2}}{2} & \text { if } N \text { is even }\end{cases}
$$

and, for any positive integer $q$,

$$
T_{R}(q, N)=R_{N} \otimes I_{q}
$$

where $I_{q}$ denotes the $q \times q$ identity matrix, $\otimes$ denotes the Kronecker product, $R_{N}=M_{N} U_{N}$ with

$$
M_{N}=\frac{1}{\sqrt{N}} \times
$$

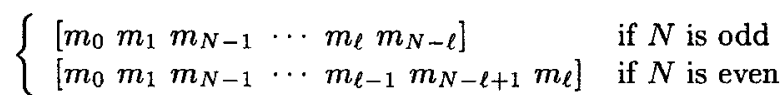

$$
U_{N}= \begin{cases}\operatorname{diag}[1 \underbrace{V V \cdots}_{\ell \ell \cdots V}] & \text { if } N \text { is odd } \\ \operatorname{diag}[1 \underbrace{V V_{\ell} \cdots V}_{\ell-1} 1] & \text { if } N \text { is even }\end{cases}
$$

and where

$$
V=\frac{1}{\sqrt{2}}\left[\begin{array}{cc}
1 & \sqrt{-1} \\
1 & -\sqrt{-1}
\end{array}\right]
$$

Then $T_{R}(q, N)$ is a real orthogonal matrix, and the following result holds.

\section{Lemma 1 Let}

$$
\mathcal{E}=\left[\begin{array}{cccc}
E & E_{0} & \cdots & E_{0} \\
E_{0} & E & \ddots & \vdots \\
\vdots & \ddots & \ddots & E_{0} \\
E_{0} & \cdots & E_{0} & E
\end{array}\right] \in \mathbb{R}^{N q \times N r}
$$

$\mathcal{F}=\operatorname{diag}\left[\begin{array}{llll}F_{1} & F_{2} & \cdots & F_{2}\end{array}\right] \in \mathbb{R}^{N q \times N_{T}}, \quad F_{1}, F_{2} \in \mathbb{R}^{q \times r}$

$E, E_{0} \in \mathbb{R}^{q \times r}$ similar construction.

$$
\begin{gathered}
\frac{1}{\sqrt{N}} \sum_{k=0}^{N-1} v_{j-1}^{k}\left(\frac{1}{\sqrt{N}}\left(E+(N-1) E_{0}\right)\right)= \\
E+(N-1) E_{0} \quad \text { if } \quad i=j=1
\end{gathered}
$$

In other words,

$$
\begin{aligned}
& \left(M_{N} \otimes I_{q}\right)^{H} \mathcal{E}\left(M_{N} \otimes I_{r}\right)= \\
& \quad \operatorname{diag}\left[\begin{array}{llll}
E+(N-1) E_{0} & E-E_{0} & \cdots & E-E_{0}
\end{array}\right]
\end{aligned}
$$

Thus,

$$
\begin{aligned}
& T_{R}^{T}(q, N) \mathcal{E} T_{R}(r, N) \\
& \quad=\left(U_{N} \otimes I_{q}\right)^{H}\left[\left(M_{N} \otimes I_{q}\right)^{H} \mathcal{E}\left(M_{N} \otimes I_{r}\right)\right]\left(U_{N} \otimes I_{r}\right) \\
& \quad=\operatorname{diag}\left[\begin{array}{llll}
E+(N-1) E_{0} & E-E_{0} & \cdots & E-E_{0}
\end{array}\right]
\end{aligned}
$$

For the case with $N$ even, the result follows through a 2. It is immediate from 1 above and the orthogonality of $T_{R}(q, N)$ and $T_{R}(r, N)$.

\section{Main Results}

1.

$$
\begin{aligned}
& T_{R}^{T}(q, N) \mathcal{E} T_{R}(r, N)= \\
& \quad \operatorname{diag}\left[\begin{array}{llll}
E+(N-1) E_{0} & E-E_{0} & \cdots & E-E_{0}
\end{array}\right]
\end{aligned}
$$

2.

$$
T_{R}(q, N) \mathcal{F} T_{R}^{T}(r, N)=\left[\begin{array}{cccc}
F & F_{0} & \cdots & F_{0} \\
F_{0} & F & \ddots & \vdots \\
\vdots & \ddots & \ddots & F_{0} \\
F_{0} & \cdots & F_{0} & F
\end{array}\right]
$$

In this section, we present the construction of the balanced realization of a symmetric composite system. Then we consider the structural properties of its truncation. To achieve these tasks, two auxiliary systems are defined with state-space realizations given by

$$
\begin{aligned}
& \left(A_{\alpha}, B_{\alpha}, C_{\alpha}\right) \equiv \\
& \quad\left(A+(N-1) A_{0}, B+(N-1) B_{0}, C+(N-1) C_{0}\right) \\
& \left(A_{\beta}, B_{\beta}, C_{\beta}\right) \equiv\left(A-A_{0}, B-B_{0}, C-C_{0}\right)
\end{aligned}
$$


It is known that $(\mathcal{A}, \mathcal{B}, \mathcal{C})$ is a minimal realization if and only if $\left(A_{\alpha}, B_{\alpha}, C_{\alpha}\right)$ and $\left(A_{\beta}, B_{\beta}, C_{\beta}\right)$ are minimal realizations . Moreover, $\mathcal{A}$ is asymptotically stable if and only if $A_{\alpha}$ and $A_{\beta}$ are asymptotically stable [Lun86].

\subsection{Balanced Realization}

Let $P_{i}, Q_{i}$ be respectively the controllability and observability grammian of the realization $\left(A_{i}, B_{i}, C_{i}\right)$ where $i=\alpha$ or $\beta$. They satisfy

$$
\begin{aligned}
& A_{i} P_{i}+P_{i} A_{i}^{T}+B_{i} B_{i}^{T^{T}}=0 \\
& A_{i}^{T} Q_{i}+Q_{i} A_{i}+C_{i}^{T} C_{i}=0
\end{aligned}
$$

The Hankel singular values of $\left(A_{i}, B_{i}, C_{i}\right)$, given by the positive square root of the eigenvalues of $P_{i} Q_{i}$, are ordered as follows

$$
\sigma_{i_{1}} \geq \sigma_{i_{2}} \geq \cdots \geq \sigma_{i_{n}}>0
$$

It is shown in [Glo84] that if $R_{i}$ is a Cholesky factor of $Q_{i}$, that is

$$
Q_{i}=R_{i}^{T} R_{i}
$$

then a singular value decomposition on $R_{i} P_{i} R_{i}^{T}$ gives

$$
U_{i} \Sigma_{i}^{2} U_{i}^{T}
$$

where $U_{i}$ is orthogonal. The balancing transformation $T_{i}$ of $\left(A_{i}, B_{i}, C_{i}\right)$, such that

$$
\left(\bar{A}_{i}, \bar{B}_{i}, \bar{C}_{i}\right)=\left(T_{i} A_{i} T_{i}^{-1}, T_{i} B_{i}, C_{i} T_{i}^{-1}\right)
$$

is a (ordered) balanced realization, is given by

$$
T_{i}=\Sigma_{i}^{-1 / 2} U_{i}^{T} R_{i}
$$

where

$$
\Sigma_{i}=\operatorname{diag}\left[\begin{array}{llll}
\sigma_{i_{1}} & \sigma_{i_{2}} & \cdots & \sigma_{i_{n}}
\end{array}\right]
$$

The balanced system has controllability and observability grammians both equal to $\Sigma_{i}$ which satisfies

$$
\begin{aligned}
& \bar{A}_{i} \Sigma_{i}+\Sigma_{i} \bar{A}_{i}^{T}+\bar{B}_{i} \bar{B}_{i}^{T}=0 \\
& \bar{A}_{i}^{T} \Sigma_{i}+\Sigma_{i} \bar{A}_{i}+\bar{C}_{i}^{T} \bar{C}_{i}=0
\end{aligned}
$$

In general, a balanced realization does not require the Hankel singular values to be ordered in $\Sigma_{i}$.

The following theorem provides an explicit way for constructing a balanced realization of the composite system $(\mathcal{A}, \mathcal{B}, \mathcal{C})$ based on the balanced realizations of $\left(A_{\alpha}, B_{\alpha}, C_{\alpha}\right)$ and $\left(A_{\beta}, B_{\beta}, C_{\beta}\right)$.

Theorem $1 A$ balanced realization of $(\mathcal{A}, \mathcal{B}, \mathcal{C})$ is given by

$$
(\overline{\mathcal{A}}, \overline{\mathcal{B}}, \overline{\mathcal{C}}) \equiv\left(\Sigma^{-1 / 2} \widetilde{A} \Sigma^{1 / 2}, \Sigma^{-1 / 2} \widetilde{B}, \widetilde{C} \Sigma^{1 / 2}\right)
$$

where

$$
\tilde{A}=\left[\begin{array}{cccc}
\bar{A} & \bar{A}_{0} & \ldots & \bar{A}_{0} \\
\bar{A}_{0} & \bar{A} & \ddots & \vdots \\
\vdots & \ddots & \ddots & \bar{A}_{0} \\
\bar{A}_{0} & \cdots & \bar{A}_{0} & \bar{A}
\end{array}\right]
$$

$$
\begin{aligned}
& \widetilde{B}=\left[\begin{array}{cccc}
\bar{B} & \bar{B}_{0} & \cdots & \bar{B}_{0} \\
\bar{B}_{0} & \bar{B} & \ddots & \vdots \\
\vdots & \ddots & \ddots & \bar{B}_{0} \\
\bar{B}_{0} & \cdots & \bar{B}_{0} & \bar{B}
\end{array}\right] \\
& \tilde{C}=\left[\begin{array}{cccc}
\bar{C}_{0} & \bar{C}_{0} & \cdots & \bar{C}_{0} \\
\bar{C}_{0} & \bar{C} & \ddots & \vdots \\
\vdots & \ddots & \ddots & \bar{C}_{0} \\
\bar{C}_{0} & \cdots & \bar{C}_{0} & \bar{C}
\end{array}\right]
\end{aligned}
$$

with

$$
\begin{array}{lll}
\bar{A}=\frac{1}{N}\left[\bar{A}_{\alpha}+(N-1) \bar{A}_{\beta}\right], & \bar{A}_{0}=\frac{1}{N}\left[\bar{A}_{\alpha}-\bar{A}_{\beta}\right] \\
\bar{B}=\frac{1}{N}\left[\bar{B}_{\alpha}+(N-1) \bar{B}_{\beta}\right], & \bar{B}_{0}=\frac{1}{N}\left[\bar{B}_{\alpha}-\bar{B}_{\beta}\right] \\
\bar{C}=\frac{1}{N}\left[\bar{C}_{\alpha}+(N-1) \bar{C}_{\beta}\right], & \bar{A}_{0}=\frac{1}{N}\left[\bar{C}_{\alpha}-\bar{C}_{\beta}\right]
\end{array}
$$

and

$$
\Sigma=\operatorname{diag}\left[\begin{array}{llll}
\Sigma_{\alpha} & \Sigma_{\beta} & \cdots & \Sigma_{\beta}
\end{array}\right]
$$

which is the controllability/observability grammian of the balanced realization.

Proof: With $P_{i}$ and $Q_{i}$ in (1) and (2), the following are defined.

$$
\begin{aligned}
\mathcal{P}: & =T_{R}(n, N) \operatorname{diag}\left[\begin{array}{llll}
P_{\alpha} & P_{\beta} & \cdots & P_{\beta}
\end{array}\right] T_{R}^{T}(n, N) \\
\mathcal{Q}: & =T_{R}(n, N) \operatorname{diag}\left[\begin{array}{llll}
Q_{\alpha} & Q_{\beta} & \cdots & Q_{\beta}
\end{array}\right] T_{R}^{T}(n, N) \\
\mathcal{R}: & =T_{R}(n, N) \operatorname{diag}\left[\begin{array}{llll}
R_{\alpha} & R_{\beta} & \cdots & R_{\beta}
\end{array}\right] T_{R}^{T}(n, N) \\
\mathcal{U}: & =T_{R}(n, N) \operatorname{diag}\left[\begin{array}{llll}
U_{\alpha} & U_{\beta} & \cdots & U_{\beta}
\end{array}\right] T_{R}^{T}(n, N)
\end{aligned}
$$

By Lemma 1, it follows that

$$
\begin{aligned}
& T_{R}^{T}(n, N)\left(\mathcal{A P}+\mathcal{P} \mathcal{A}^{T}+\mathcal{B} \mathcal{B}^{T}\right) T_{R}(n, N) \\
& =T_{R}^{T}(n, N) \mathcal{A} T_{R}(n, N) T_{R}^{T}(n, N) \mathcal{P} T_{R}(n, N) \\
& +T_{R}^{T}(n, N) \mathcal{P} T_{R}(n, N) T_{R}^{T}(n, N) \mathcal{A}^{T} T_{R}(n, N) \\
& \quad+T_{R}^{T}(n, N) \mathcal{B} T_{R}(m, N) T_{R}^{T}(m, N) \mathcal{B}^{T} T_{R}(n, N) \\
& =\operatorname{diag}\left[A_{\alpha} P_{\alpha}+P_{\alpha} A_{\alpha}^{T}+B_{\alpha} B_{\alpha}^{T}\right. \\
& A_{\beta} P_{\beta}+P_{\beta} A_{\beta}^{T}+B_{\beta} B_{\beta}^{T} \ldots \\
& \left.\quad A_{\beta} P_{\beta}+P_{\beta} A_{\beta}^{T}+B_{\beta} B_{\beta}^{T}\right] \\
& =0
\end{aligned}
$$

and hence $\mathcal{A P}+\mathcal{P} \mathcal{A}^{T}+\mathcal{B} \mathcal{B}^{T}=0$. Sirnilarly, the following equations are established

$$
\begin{aligned}
\mathcal{A}^{T} \mathcal{Q}+\mathcal{Q} \mathcal{A}+\mathcal{C}^{T} \mathcal{C} & =0 \\
\mathcal{Q} & =\mathcal{R}^{T} \mathcal{R} \\
\mathcal{R} \mathcal{P} \mathcal{R}^{T} & =\mathcal{U} \Sigma^{2} \mathcal{U}^{T}
\end{aligned}
$$

Thus, it is easy to construct a balancing transformation

$$
\begin{aligned}
\mathcal{T}:= & \Sigma^{-1 / 2} \mathcal{U}^{T} \mathcal{R} \\
= & \operatorname{diag}\left[\begin{array}{llll}
\Sigma_{\alpha}^{-1 / 2} & \Sigma_{\beta}^{-1 / 2} & \cdots & \Sigma_{\beta}^{-1 / 2}
\end{array}\right] T_{R}(n, N) \\
& \quad \times \operatorname{diag}\left[\begin{array}{lllll}
U_{\alpha}^{T} R_{\alpha} & U_{\beta}^{T} R_{\beta} & \cdots & U_{\beta}^{T} R_{\beta}
\end{array}\right] T_{R}^{T}(n, N)
\end{aligned}
$$

which gives

$$
\mathcal{T} \mathcal{P} \mathcal{T}^{T}=\Sigma \quad, \quad\left(\mathcal{T}^{T}\right)^{-1} \mathcal{Q} \mathcal{T}^{-1}=\Sigma
$$




$$
\begin{aligned}
\mathcal{T} \mathcal{A} \mathcal{T}^{-1} \Sigma+\Sigma\left(\mathcal{T}^{T}\right)^{-1} \mathcal{A}^{T} \mathcal{T}^{T}+\mathcal{T} B \mathcal{B}^{T} \mathcal{T}^{T} & =0 \\
\left(\mathcal{T}^{T}\right)^{-1} \mathcal{A}^{T} \mathcal{T}^{T} \Sigma+\Sigma \mathcal{T} \mathcal{A} \mathcal{T}^{-1}+\left(\mathcal{T}^{T}\right)^{-1} \mathcal{C}^{T} \mathcal{C} \mathcal{T}^{-1} & =0
\end{aligned}
$$

In other words, $\left(\mathcal{T} \mathcal{A} \mathcal{T}^{-1}, \mathcal{T B}, \mathcal{C} \mathcal{T}^{-1}\right)$ is a balanced realization. Now, it is a straightforward algebraic manipulation to establish that

$\mathcal{T} \mathcal{A} \mathcal{T}^{-1}=\Sigma^{-1 / 2} \widetilde{A} \Sigma^{1 / 2} \quad, \quad \mathcal{T} \mathcal{B}=\Sigma^{-1 / 2} \widetilde{B} \quad, \quad \mathcal{C} \mathcal{T}^{-1}=\widetilde{C} \Sigma^{1 / 2}$

Hence, the result follows.

Remark 1 The result in Theorem 1 indicated that to construct a balanced realization of a symmetric composite system $(\mathcal{A}, \mathcal{B}, \mathcal{C})$ of order $N n$ amounts to the balancing of two lower order auxiliary systems $\left(A_{\alpha}, B_{\alpha}, C_{\alpha}\right)$ and $\left(A_{\beta}, B_{\beta}, C_{\beta}\right)$, both of order $n$. The reduction in computation is especially significant if $N$ is large.

Remark 2 The grammian $\Sigma$ of the balanced realization $(\overline{\mathcal{A}}, \overline{\mathcal{B}}, \overline{\mathcal{C}})$ is not ordered in terms of the magnitude of the Hankel singular values which compose of those from $\left(A_{\alpha}, B_{\alpha}, C_{\alpha}\right)$ and $\left(A_{\beta}, B_{\beta}, C_{\beta}\right)$ (repeated $(N-1)$ times). It is also observed that the balanced realization $(\overline{\mathcal{A}}, \overline{\mathcal{B}}, \overline{\mathcal{C}})$ is not a symmetric composite system as defined, though its structure still has a kind of symmetry. In fact, it represents an external system connected symmetrically to identical subsystems which are themselves connected symmetrically. Such class of systems has been studied by Yang and Zhang [YZ95b] for their structural properties. On the other hand, it is interesting to realize that the system with realization $(\widetilde{A}, \widetilde{B}, \widetilde{C})$, also having a symmetric composite structure, is similar to the balanced realization $(\overline{\mathcal{A}}, \overline{\mathcal{B}}, \overline{\mathcal{C}})$ via a diagonal transformation. That is, $(\overline{\mathcal{A}}, \overline{\mathcal{B}}, \overline{\mathcal{C}})$ is also a balanced realization of $(\widetilde{A}, \widetilde{B}, \widetilde{C})$ with $\Sigma^{-1 / 2}$ as the balancing transformation. This fact is especially important when forming the balanced reduced models. In the very special case when $\Sigma_{\alpha}=\Sigma_{\beta}$, the balanced realization $(\overline{\mathcal{A}}, \overline{\mathcal{B}}, \overline{\mathcal{C}})$ is also a symmetric composite system.

\subsection{Model Reduction by Truncation}

Let $\lambda(\cdot)$ denote the spectrum of the matrix $(\cdot)$. With $i=$ $\alpha$ or $\beta$, partition $\Sigma_{i}$ such that

$$
\Sigma_{i}=\operatorname{diag}\left[\begin{array}{ll}
\Sigma_{i_{1}} & \Sigma_{i_{2}}
\end{array}\right]
$$

where $\Sigma_{i_{1}} \in \mathbb{R}^{k_{i} \times k_{i}}\left(k_{i} \geq 0\right)$ under that condition that

$$
\lambda\left(\Sigma_{i_{1}}\right) \cap \lambda\left(\Sigma_{i_{2}}\right)=\emptyset
$$

Additionally, we require that

$$
\begin{aligned}
& \lambda\left(\Sigma_{\alpha_{1}}\right) \cap \lambda\left(\Sigma_{\beta_{2}}\right)=\emptyset \\
& \lambda\left(\Sigma_{\alpha_{2}}\right) \cap \lambda\left(\Sigma_{\beta_{1}}\right)=\emptyset
\end{aligned}
$$

For model reduction based on a balanced truncation, the Hankel singular values in $\Sigma_{\alpha_{2}}$ and $\Sigma_{\beta_{2}}$ are those amongst the least in $\Sigma$ (that is, the minimum Hankel singular value amongst $\Sigma_{\alpha_{1}}$ and $\Sigma_{\beta_{1}}$ is strictly greater than the largest Hankel singular value amongst $\Sigma_{\alpha_{2}}$ and $\Sigma_{\beta_{2}}$ ). In this case, the order of the reduced model is $k_{\alpha}+(N-1) k_{\beta}$.
For $i=\alpha$ or $\beta$, we partition the balanced realization $\left(\bar{A}_{i}, \bar{B}_{i}, \bar{C}_{i}\right)$ as follows:

$$
\begin{aligned}
\bar{A}_{i} & =\left[\begin{array}{ll}
\bar{A}_{i_{11}}^{1} & \bar{A}_{i_{12}}^{1} \\
\bar{A}_{i_{21}}^{1} & \bar{A}_{i_{22}}^{1}
\end{array}\right]=\left[\begin{array}{ll}
\bar{A}_{i_{11}}^{2} & \bar{A}_{i_{12}}^{2} \\
\bar{A}_{i_{21}}^{2} & \bar{A}_{i_{22}}^{2}
\end{array}\right] \\
& =\left[\begin{array}{ll}
\bar{A}_{i_{11}}^{3} & \bar{A}_{i_{12}}^{3} \\
\bar{A}_{i_{21}}^{3} & \bar{A}_{i_{22}}^{3}
\end{array}\right]=\left[\begin{array}{ll}
\bar{A}_{i_{11}}^{4} & \bar{A}_{i_{12}}^{4} \\
\bar{A}_{i_{21}}^{4} & \bar{A}_{i_{22}}^{4}
\end{array}\right]
\end{aligned}
$$

with $\bar{A}_{i_{11}}^{1} \in \mathbb{R}^{k_{\alpha} \times k_{\alpha}}, \bar{A}_{i_{11}}^{2} \in \mathbb{R}^{k_{\beta} \times k_{\beta}}, \bar{A}_{i_{11}}^{3} \in \mathbb{R}^{k_{\alpha} \times k_{\beta}}$, $\bar{A}_{i_{11}}^{4} \in \mathbb{R}^{k_{\beta} \times k_{\alpha}}$,

$$
\bar{B}_{i}=\left[\begin{array}{l}
\bar{B}_{i_{1}}^{1} \\
\bar{B}_{i_{2}}^{1}
\end{array}\right]=\left[\begin{array}{l}
\bar{B}_{i_{1}}^{2} \\
\bar{B}_{i_{2}}^{2}
\end{array}\right]
$$

with $\bar{B}_{i_{1}}^{1} \in \mathbb{R}^{k_{\alpha} \times m}, \bar{B}_{i_{1}}^{2} \in \mathbb{R}^{k_{\beta} \times m}$

$$
\bar{C}_{i}=\left[\begin{array}{ll}
\bar{C}_{i_{1}}^{1} & \bar{C}_{i_{2}}^{1}
\end{array}\right]=\left[\begin{array}{ll}
\bar{C}_{i_{1}}^{2} & \bar{C}_{i_{2}}^{2}
\end{array}\right]
$$

with $\bar{C}_{i_{1}}^{1} \in \mathbb{R}^{p \times k_{\alpha}}, \bar{C}_{i_{1}}^{2} \in \mathbb{R}^{p \times k_{\beta}}$.

Then we have the following theorem which gives an explicit construction of the balanced reduced-order models.

Theorem 2 With the notation employed, by truncating the states of the balanced realization $(\overline{\mathcal{A}}, \overline{\mathcal{B}}, \overline{\mathcal{C}})$ associated with the Hankel singular values in $\Sigma_{\alpha_{2}}$ and $\Sigma_{\beta_{2}}$ under conditions (3), (4), and (5), a reduced-order balanced realization of order $k_{\alpha}+(N-1) k_{\beta}$ is given by

$$
(\widehat{\mathcal{A}}, \widehat{\mathcal{B}}, \widehat{\mathcal{C}}) \equiv\left(\widehat{\Sigma}^{-1 / 2} \widehat{A}^{1 / 2}, \widehat{\Sigma}^{-1 / 2} \widehat{B}, \widehat{C} \widehat{\Sigma}^{1 / 2}\right)
$$

where

$$
\begin{aligned}
\widehat{\Sigma} & =\operatorname{diag}\left[\begin{array}{cccc}
\Sigma_{\alpha_{1}} & \Sigma_{\beta_{1}} & \cdots & \Sigma_{\beta_{1}}
\end{array}\right] \\
\widehat{A} & =\left[\begin{array}{ccccc}
\widehat{A}_{0} & \widehat{A}_{01} & \widehat{A}_{01} & \cdots & \widehat{A}_{01} \\
\widehat{A}_{10} & \widehat{A}_{1} & \widehat{A}_{11} & \cdots & \widehat{A}_{11} \\
\widehat{A}_{10} & \widehat{A}_{11} & \widehat{A}_{1} & \ddots & \vdots \\
\vdots & \vdots & \ddots & \ddots & \widehat{A}_{11} \\
\widehat{A}_{10} & \widehat{A}_{11} & \cdots & \widehat{A}_{11} & \widehat{A}_{1}
\end{array}\right] \\
\widehat{B} & =\left[\begin{array}{ccccc}
\widehat{B}_{0} & \widehat{B}_{01} & \widehat{B}_{01} & \cdots & \widehat{B}_{01} \\
\widehat{B}_{11} & \widehat{B}_{1} & \widehat{B}_{11} & \cdots & \widehat{B}_{11} \\
\widehat{B}_{11} & \widehat{B}_{11} & \widehat{B}_{1} & \ddots & \vdots \\
\vdots & \vdots & \ddots & \ddots & \widehat{B}_{11} \\
\widehat{B}_{11} & \widehat{B}_{11} & \cdots & \widehat{B}_{11} & \widehat{B}_{1}
\end{array}\right] \\
\widehat{C} & =\left[\begin{array}{ccccc}
\widehat{C}_{0} & \widehat{C}_{11} & \widehat{C}_{11} & \cdots & \widehat{C}_{11} \\
\widehat{C}_{10} & \widehat{C}_{1} & \widehat{C}_{11} & \cdots & \widehat{C}_{11} \\
\widehat{C}_{10} & \widehat{C}_{11} & \widehat{C}_{1} & \ddots & \vdots \\
\vdots & \vdots & \ddots & \ddots & \widehat{C}_{11} \\
\widehat{C}_{10} & \widehat{C}_{11} & \cdots & \widehat{C}_{11} & \widehat{C}_{1}
\end{array}\right]
\end{aligned}
$$

with

$$
\begin{aligned}
\widehat{A}_{0} & =\frac{1}{N}\left[\bar{A}_{\alpha_{11}}^{1}+(N-1) \bar{A}_{\beta_{11}}^{1}\right] \\
\widehat{A}_{01} & =\frac{1}{N}\left[\bar{A}_{\alpha_{11}}^{3}-\bar{A}_{\beta_{11}}^{3}\right] \\
\hat{A}_{10} & =\frac{1}{N}\left[\bar{A}_{\alpha_{11}}^{4}-\bar{A}_{\beta_{11}}^{4}\right]
\end{aligned}
$$




$$
\begin{aligned}
\widehat{A}_{1} & =\frac{1}{N}\left[\bar{A}_{\alpha_{11}}^{2}+(N-1) \bar{A}_{\beta_{11}}^{2}\right] \\
\widehat{A}_{11} & =\frac{1}{N}\left[\bar{A}_{\alpha_{11}}^{2}-\bar{A}_{\beta_{11}}^{2}\right] \\
\widehat{B}_{0} & =\frac{1}{N}\left[\bar{B}_{\alpha_{1}}^{1}+(N-1) \bar{B}_{\beta_{1}}^{1}\right] \\
\widehat{B}_{01} & =\frac{1}{N}\left[\bar{B}_{\alpha_{1}}^{1}-\bar{B}_{\beta_{1}}^{1}\right] \\
\widehat{B}_{1} & =\frac{1}{N}\left[\bar{B}_{\alpha_{1}}^{2}+(N-1) \bar{B}_{\beta_{1}}^{2}\right] \\
\widehat{B}_{11} & =\frac{1}{N}\left[\bar{B}_{\alpha_{1}}^{2}-\bar{B}_{\beta_{1}}^{2}\right] \\
\widehat{C}_{0} & =\frac{1}{N}\left[\bar{C}_{\alpha_{1}}^{1}+(N-1) \bar{C}_{\beta_{1}}^{1}\right] \\
\widehat{C}_{10} & =\frac{1}{N}\left[\bar{C}_{\alpha_{1}}^{1}-\bar{C}_{\beta_{1}}^{1}\right] \\
\widehat{C}_{1} & =\frac{1}{N}\left[\bar{C}_{\alpha_{1}}^{2}+(N-1) \bar{C}_{\beta_{1}}^{2}\right] \\
\widehat{C}_{11} & =\frac{1}{N}\left[\bar{C}_{\alpha_{1}}^{2}-\bar{C}_{\beta_{1}}^{2}\right]
\end{aligned}
$$

Proof: Based on Theorem 1 and the construction of $(\widehat{\mathcal{A}}, \widehat{\mathcal{B}}, \widehat{\mathcal{C}})$, the result is essentially obtained by deleting the appropriate columns and rows in the balanced realization $(\mathcal{A}, \mathcal{B}, \mathcal{C})$.

Similar to Theorem $1,(\widehat{A}, \widehat{B}, \widehat{C})$ is in general not a symmetric composite system. Furthermore, although $(\widehat{A}, \widehat{B}, \widehat{C})$ is not a balanced realization in general, it gives a truncated balanced realization model since it is similar to $(\widehat{\mathcal{A}}, \widehat{\mathcal{B}}, \widehat{\mathcal{C}})$. Two interesting situations are when the truncated realization $(\widehat{\mathcal{A}}, \widehat{\mathcal{B}}, \widehat{\mathcal{C}})$ and the realization $(\widehat{A}, \widehat{B}, \widehat{C})$ are also symmetric composite systems. The former case corresponding to a preservation of the symmetric composite structure under a balanced truncation. These are described in the following corollary which can be obtained by directly examining these realizations in Theorem 2.

\section{Corollary 1}

1. If $k_{\alpha}=0$, then $(\widehat{\mathcal{A}}, \widehat{\mathcal{B}}, \widehat{\mathcal{C}})$ is a symmetric composite system.

2. If $k_{\alpha}=k_{\beta}$, then $(\widehat{A}, \widehat{B}, \widehat{C})$ is a symmetric composite system.

Remark 3 We can also see that $(\widehat{\mathcal{A}}, \widehat{\mathcal{B}}, \widehat{\mathcal{C}})$ corresponds to a balanced realization of $(\widehat{A}, \widehat{B}, \widehat{C})$ with $\widehat{\Sigma}^{-1 / 2}$ as the balancing transformation.

\section{Conclusions}

We have studied the structural properties of the balanced realization of a class of symmetric composite systems. The construction of a balanced realization of such system is reduced to the balancing of two auxiliary systems which are usually of substantially lower dimensions. This leads to a major reduction in the computation effort. It was shown that the balanced realization and its truncation generate a generalized symmetric structure which under some conditions, preserve the symmetric composite structure.

\section{References}

[AC91] C. S. Araujo and J. C. Castro. Application of power system stabilisers in a plant with identical units. Proc. IEE, Pt. C, 138:11-18, 1991.

[BL88] L. Bakule and J. Lunze. Decentralized design of feedback control for large-scale systems. $K y$ bernetika, 24:1-100, 1988.

[Glo84] K. Glover. All optimal Hankel-norm approximations of linear multivariable systems and their $L^{\infty}$-error bounds. Int. J. Control, 39(6):1115-1193, 1984.

[LA92] J. Lam and B. D. O. Anderson. $L_{1}$ impulse response error bound for balanced truncation. Systems and Control Letters, 18:129-137, 1992.

[Liu92] X. Liu. Output regulation of strongly coupled symmetric composite systems. Automatica, 28:1037-1041, 1992.

[Lun86] J. Lunze. Dynamics of strongly coupled symmetric composite systems. Int. J. Control, 44:1657-1670, 1986.

[Moo81] B. C. Moore. Principal component analysis in linear systems: controllability, observability, and model reduction. IEEE Trans. Automat. Contr., AC-26(2):17-32, 1981.

[PS82] L. Pernebo and L. M. Silverman. Model reduction via balanced state space representations. IEEE Trans. Automat. Contr., AC-27(2):382$387,1982$.

[SE91] M. K. Sundareshan and R. M. Elbanna. Qualitative analysis and decentralized controller synthesis for a class of large-scale systems with symmetrically interconnected subsystems. $A u$ tomatica, 27:383-388, 1991.

[VS82] M. Vukobratovic and D. M. Stokic. Control of Manipulation Robots: Theory and Application. Springer-Verlag, Berlin, 1982.

[YZ95a] G. H. Yang and S. Zhang. Stabilizing con trollers for uncertain symmetric composite systems. Automatica, 31(2):337-340, 1995.

[YZ95b] G. H. Yang and S. Zhang. Structural properties of large-scale systems with similar structures. Automatica, 31(7):1011--1017, 1995. 